Acta Crystallographica Section B

\section{Structural}

\section{Science}

ISSN 0108-7681

J. J. Holstein, ${ }^{a}$ P. Luger, ${ }^{b}$ R. Kalinowski, ${ }^{\text {b }}$ S. Mebs, ${ }^{\text {b }}$ C. Paulman $^{\mathrm{c}} ¥$ and B. Dittrich ${ }^{\mathrm{a} *}$

alnstitut für Anorganische Chemie, GeorgAugust-Universität, Tammannstrasse 4, D37077 Göttingen, Germany, ${ }^{\mathbf{b}}$ Institut für Chemie/Kristallographie der Freien Universität Berlin, Fabeckstrasse 36a, D-14195 Berlin, Germany, and ${ }^{\mathrm{C}}$ Mineralogisch-Petrologisches Institut, Universität Hamburg, Grindelallee 48, D-20146 Hamburg, Germany

\# c/o DESY/HASYLAB, Notkestrasse 85, 22607 Hamburg, Germany.

Correspondence e-mail: bdittri@gwdg.de
(C) 2010 International Union of Crystallography Printed in Singapore - all rights reserved

\title{
Validation of experimental charge densities: refinement of the macrolide antibiotic roxithro- mycin
}

Multipole refinements of larger organic molecules have so far been limited to a few exceptional cases. We report an investigation of the detailed experimental electron-density distribution (EDD) of roxithromycin, a macrolide antibiotic consisting of 134 atoms. Although the experimental multipole refinement on high-resolution synchrotron data converged smoothly, validation of the electron density by calculation of an 'experiment minus invariom' difference density revealed conformational disorder of the $\mathrm{H}$ atoms. Hydrogen disorder is shown to affect the EDD, the electrostatic potential and atomic properties as defined by Bader's quantum theory of atoms in molecules. A procedure to obtain the electron density distribution in the presence of disorder is proposed.

\section{Introduction}

Roxithromycin is a semisynthetic antibiotic. Together with the better known erythromycin, the molecule is part of the macrolide class of compounds. Its molecular structure consists of a 14-membered lactone ring and the two sugar residues cladinose and desosamine (see Fig. 1).

Erythromycin and roxithromycin only differ in the substitution pattern of the lactone ring. In the case of roxithromycin, the substituted oxime function replaces a keto group. This increases stability under acidic conditions such as found in the stomach of the human body. Macrolide antibiotics are known to suppress protein synthesis in the 50S subunit (Harms et al., 2001) of the bacterial ribosome. They interact with nucleotides of the peptidyl transferase by blocking the tunnel that channels the nascent peptides to the peptidyl transferase center

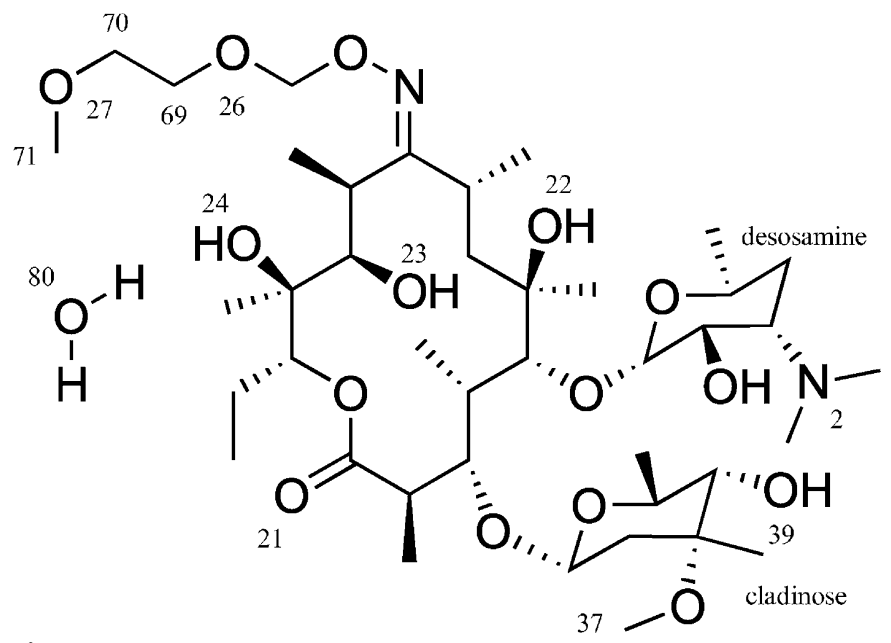

Figure 1

Structural formula of roxithromycin with numbering scheme for $\mathrm{O}$ atoms involved in hydrogen bonding and $\mathrm{C}$ atoms carrying disordered $\mathrm{H}$ atoms.
Received 22 October 2009

Accepted 5 July 2010 
(Schlünzen et al., 2001). Owing to their biological function and emerging bacterial resistance (Tu et al., 2005), the detailed electronic density distribution (EDD) $\rho(\mathbf{r})$ is of special interest for these compounds.

X-ray single-crystal structure analysis was crucial in identifying the chemical composition of macrolide antibiotics; the macrolide structure of a heavy-atom derivative of erythromycin was first solved in 1965 by Harris et al. (1965); the structure of roxithromycin monohydrate was first reported in 1988 by Bachet et al. (1988). ${ }^{\mathbf{1}}$ A primary objective of this study is to extract a physicochemically plausible EDD for roxithromycin molecules in the crystal lattice from high-resolution Bragg data. Properties like the electrostatic potential (ESP) and the dipole moment can then be extracted from the EDD. Both properties are believed to be highly relevant in drugreceptor recognition processes. Our investigation is part of a systematic study on the biological function of the macrolide antibiotics on the level of their EDD.

It has been emphasized that the methodology of chargedensity modeling has come of age (Coppens, 2005). Nevertheless, we believe that a number of minor, but still problematic, issues remain. In our opinion, treatment of the electron density of $\mathrm{H}$ atoms is one of those issues (Abramov et al., 2000). It is well known that the scattering of $\mathrm{H}$ atoms is weak and limited in reciprocal space when compared with e.g. carbon. Since an estimation of the anisotropic displacement parameters (ADPs) of $\mathrm{H}$ atoms is now routinely possible (Roversi \& Destro, 2004; Whitten \& Spackman, 2006; Madsen, 2006; Munshi et al., 2008), finer details on the EDD of $\mathrm{H}$ atoms can now be experimentally investigated. Hybrid scattering factors for $\mathrm{H}$ atoms (see \$4.2) might be a further improvement.

Differences between the EDD of a molecule in the crystal and its gas-phase counterpart (with the same geometry and conformation) are mainly due to hydrogen bonding, but are also caused by crystal packing and the crystal field (Gatti et al., 1994). Since these differences were found to be small when 'projected' onto a multipole model in situations where disorder is absent (Spackman et al., 1999; Dittrich \& Spackman, 2007), the experimental and ab initio ESPs are expected to agree qualitatively. Combining theory and experiment can therefore help to identify problematic parts of a structural model, and to assess how well the additional information content on packing and hydrogen bonding has indeed been extracted and modeled from the Bragg data within the accuracy of the multipole model. Purely theoretical approaches such as periodic Hartree-Fock or density functional theory computations unfortunately have limitations in their applicability to larger systems like roxithromycin. For this reason, database approaches (Zarychta et al., 2007; Dittrich et al., 2006; Volkov et al., 2007) have been developed to improve the scattering model for larger molecules, in which the total electron density of a molecule is reconstructed from electron-density fragments (pseudoatoms). The transferability of pseudoatom density fragments has been verified experi-

\footnotetext{
${ }^{1}$ Although the determination of the absolute configuration was a crucial part in this paper, the published structure has the wrong absolute configuration.
}

Table 1

Crystal and structure refinement data for roxithromycin.

\begin{tabular}{ll}
\hline Crystal data & \\
Chemical formula & $\mathrm{C}_{41} \mathrm{H}_{76} \mathrm{~N}_{2} \mathrm{O}_{15} \cdot 0.88 \mathrm{H}_{2} \mathrm{O}$ \\
$M_{r}$ & 852.96 \\
Crystal system, space group & Orthorhombic, $P 2_{1} 2_{1} 2_{1}$ \\
$T(\mathrm{~K})$ & 100 \\
$a, b, c(\AA)$ & $11.624(2), 16.748(3), 24.018(5)$ \\
$V\left(\AA^{3}\right)$ & $4675.8(15)$ \\
$Z$ & 4 \\
Radiation type & Synchrotron, $\lambda=0.5600 \AA$ \\
$\mu\left(\mathrm{mm}^{-1}\right)$ & 0.06 \\
Crystal size $(\mathrm{mm})$ & $0.50 \times 0.35 \times 0.30$ \\
& \\
Data collection & Huber Kappa diffractometer with \\
Diffractometer & MAR165 CCD detector \\
& $474571,28457,26324$ \\
No. of measured, independent and & \\
$\quad$ observed $[F>3 \sigma F]$ reflections & 99.6 \\
Completeness $(\%)$ & 0.034 \\
$R_{\text {int }}$ & \\
Refinement & See Table 2 \\
\hline
\end{tabular}

$R_{\mathrm{int}}\left(F^{2}\right)=\sum \mid F_{\mathrm{o}}^{2}-F_{\mathrm{o}}^{2}($ mean) $) \mid / \sum F_{\mathrm{o}}^{2}$.

mentally in various studies (Rödel et al., 2006; Luger, 2007; Grabowsky et al., 2009). Known advantages of database approaches involve rapid property calculation and a more accurate experimental geometry that can also be obtained for data of normal resolution. Databases can also contribute to another aspect of charge-density research that requires further study: electron-density validation. Concerning the validity of a particular multipole model, we focus here on hydrogen disorder that can potentially bias the least-squares refinement parameters, and investigate it by exploiting the interplay between experiment and theory (Coppens \& Volkov, 2004).

\section{Experimental}

A crystal of the title compound was selected from the commercially obtained (Sigma Aldrich) sample. The X-ray diffraction experiment was carried out on a $\kappa$-axis diffractometer equipped with a MAR165 CCD detector using an Oxford Cryosystems nitrogen-gas-stream cooling device at beamline F1 of HASYLAB/DESY in Hamburg, Germany. The wavelength chosen was $\lambda=0.5600 \AA$. The data collection strategy consisted of four runs, two runs with $2 \theta=0^{\circ}$ and two runs with $2 \theta=-40^{\circ}$. The detector-to-crystal distance was $67 \mathrm{~mm}$ and the frames covered $1^{\circ}\left(2 \theta=0^{\circ}\right)$ or $1.5^{\circ}\left(2 \theta=-40^{\circ}\right)$ in $\varphi$.

Thanks to good crystal quality and a strong primary beam intensity, a maximum resolution of $\sin \theta / \lambda=1.25 \AA^{-1}$ was reached. However, closer inspection of the data in terms of the $I / \sigma$ ratio, $R_{\text {int }}$ and redundancy of the higher-resolution shells convinced us to cut the data at $d=0.45 \AA\left(\sin \theta / \lambda=1.11 \AA^{-1}\right)$ - still a handsome resolution for a structure of this size. Integration and scaling were performed with the $X D S$ software (Kabsch, 1993) and unit-cell dimensions were averaged using CELLPARM. Merging was performed with XPREP 
(Bruker AXS). Further crystallographic details can be found in Table 1 and in the supplementary information. ${ }^{2}$

\section{Charge density model, refinement strategy}

The previously deposited structure of roxithromycin (Bachet et al., 1988) had incorrect handedness (CIF code: FUXYOM). Therefore, the structure was solved again with direct methods using SHELXS. SHELXL97 (Sheldrick, 2008) was then employed for an initial promolecule refinement, in which all $78 \mathrm{H}$ atoms were located by difference-Fourier synthesis; the solvent water molecule was found to be only partially occupied $(88 \%)$. This occupation was kept fixed in further refinements. For all subsequent aspherical-atom refinements, the multipole formalism by Hansen \& Coppens (1978) as implemented in the program package $X D$ (Koritsánszky et al., 2003) was used. The modeling of the EDD of roxithromycin was initiated with an invariom refinement. The preprocessor program InvariomTool (Hübschle et al., 2007) was used to generate an initial master and input file for $X D$ by a transfer of multipole populations up to hexadecapolar level, including the assignment of $\kappa$ parameters. After this 'invariom transfer', coordinates and displacement parameters (anisotropic for non- $\mathrm{H}$ atoms, isotropic for $\mathrm{H}$ atoms) were refined to convergence, while initially fixing multipole parameters to invariom database values (Dittrich et al., 2006). Bond distances to $\mathrm{H}$ atoms were set to the values from quantum chemical geometry optimizations of the same model compounds, which were also used to extract the invarioms. ${ }^{3}$ The procedure chosen has been shown to allow deconvolution of ADPs from electron density (Dittrich et al., 2008). To estimate the ADPs for $\mathrm{H}$ atoms, deconvoluted non-hydrogen ADPs from the invariom model were used for a TLS refinement (Schomaker \& Trueblood, 1968) to calculate the contribution of rigid-body motion in roxithromycin via utilization of the SHADE2 server and the program THMA11 (Madsen et al., 2004; Madsen, 2006). Four groups in the molecule were assumed to behave like rigid bodies: each of the two sugars, the macrolide ring and the oxime side chain. With the resulting anisotropic description of all atoms, the invariom refinement was repeated, providing starting values for all subsequent refinements. This refinement is referred to as $\kappa$-restricted invariom refinement (IR). Only three $\mathrm{C}-\mathrm{O}$ single bonds are involved in hydrogen disorder (see below); all of the other bonds pass the Hirshfeld test (Hirshfeld, 1976) (see supplementary information) in the IR, indicating a proper deconvolution of electron density and thermal motion. In the next step, we performed an experimental multipole refinement (EMR) in which multipole parameters were adjusted against the measured data. In order

\footnotetext{
${ }^{2}$ Supplementary data for this paper are available from the IUCr electronic archives (Reference: GW5005). Services for accessing these data are described at the back of the journal.

${ }^{3}$ These $X-\mathrm{H}$ distances are in close agreement with the values from neutron diffraction experiments (Allen \& Bruno, 2010), but are more specific since a finer distinction between different chemical environments is possible in the invariom formalism, whereas neutron distances are averaged over a larger number of samples with different chemical environments considered to be similar.
}

Table 2

Figures of merit for the three different refinement models of roxithromycin: invariom refinement (IR), experimental multipole refinement (EMR) and mixed model refinement (MMR), further details in the text.

\begin{tabular}{llll}
\hline FOM & IR & EMR & MMR \\
\hline Parameters & 532 & 725 & 850 \\
Reflections & 6324 & 26324 & 26324 \\
$R(F)$ & 0.0221 & 0.0204 & 0.0201 \\
$w R(F)$ & 0.0190 & 0.0167 & 0.0168 \\
$R_{\text {all }}(F)$ & 0.0246 & 0.0229 & 0.0226 \\
GoF & 4.03 & 3.54 & 3.59 \\
$\Delta \rho_{\min }, \Delta \rho_{\max }\left(\mathrm{e}^{-3}\right)$ & $-0.30,0.33$ & $-0.33,0.32$ & $-0.20,0.20$ \\
\hline
\end{tabular}

$w R_{w}(F)=\left[\left.\sum w|| F_{\mathrm{o}}|-k| F_{\mathrm{c}}\right|^{2} / \sum w\left|F_{\mathrm{o}}\right|^{2}\right]^{1 / 2}, w=1 / \sigma^{2}, R_{1}(F)=\sum|| F_{\mathrm{o}}|-| F_{\mathrm{c}}|| / \sum\left|F_{\mathrm{o}}\right|$, $R_{\text {all }}(F)$ using all reflections, $\mathrm{GoF}=\left[\left(\sum\left\|F_{\mathrm{o}}|-k| F_{\mathrm{c}}\right\|^{2}\right) /\left(n_{\mathrm{o}}-m_{\mathrm{var}}\right)\right]^{1 / 2}, \Delta \rho$ is the residual density.

to reduce the number of multipole parameters to be refined, pseudoatoms with the same sphere of nearest neighbors ${ }^{4}$ were considered to be chemically equivalent, i.e. chemical constraints were used when the same invariom had been assigned in the IR model. To make the multipole model more flexible, chemical constraints were released for those $\mathrm{H}$ atoms involved in hydrogen bonding. Furthermore, local atomic site symmetry analogous to the IR model was applied. The order of the multipole expansion of non- $\mathrm{H}$ atoms was four, the hexadecapolar level. Coordinates of all atoms and ADPs of $\mathrm{H}$ atoms from a previous invariom refinement were kept, thereby also enabling a direct comparison of the refined electron density with the invariom prediction on a grid. Additionally, all refinements were performed with fixed $\kappa$ parameters, in analogy to the $\kappa^{\prime}$ restricted multipole model, which had been proposed to avoid basis-set overlap errors in experimental multipole refinements (Abramov et al., 1999; Volkov et al., 2001) especially for larger structures. Special care was taken to model the electron density of the $\mathrm{H}$ atoms by comparing two multipole models. Treatment with hybrid scattering factors where higher multipoles $\left(l_{\max } \geq 2\right)$ were kept at the database result and only monopoles and dipoles were refined for $\mathrm{H}$ atoms proved superior to a conventional treatment with $l_{\max }=1 .{ }^{5}$ Our final multipole model that takes into account findings described in $\$ 4.1$ will be introduced and explained there. Figures of merit for the three different refinement models can be found in Table 2 .

\section{Results and discussion}

The molecular structure of roxithromycin together with the solvent water molecule is displayed in an ORTEP representation (Burnett \& Johnson, 1996) generated with PLATON (Spek, 2003) in Fig. 2.

\footnotetext{
${ }^{4}$ For $\mathrm{H}$ atoms a sphere of next-nearest neighbors defined whether they were considered chemically equivalent or not.

${ }^{5}$ Details from the comparison of these two models and implications on electron density modeling are given in $\$ 4.2$.
} 


\subsection{Validation of $\rho(r)$ allows the detection of dynamic disorder}

Empirical evidence shows that crystal quality is often inversely proportional to the size of a molecule under study. This might be partly attributed to the higher conformational flexibility of larger molecules, which is often linked to the occurrence of disorder and/or increased mosaic spread, providing a reason why charge-density analyses of molecules containing more than 100 atoms are rare to date.

To identify disordered sites and to validate the experimental electron density with the theoretical database expectation values, we make use of a difference-density calculation by comparing experimental multipole and invariom refinements. Difference densities have proven to be useful before. Not mentioning decades of deformation-density mapping, difference-density calculations were recently used to validate the population parameters of the invariom database using experimental data (Dittrich et al., 2007) and to compare the University of Buffalo Database UBDB (Dominiak et al., 2007) with the invariom database (Johnas et al., 2009). Even though

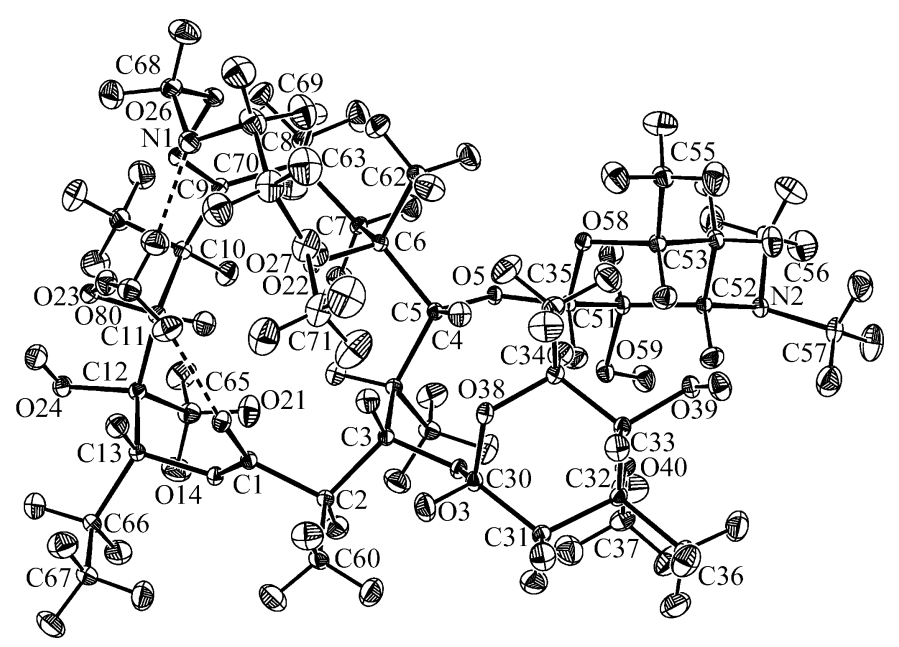

Figure 2

Asymmetric unit of roxithromycin including hydrogen bonds (dashed). ADPs for all atoms are at the $30 \%$ probability level.

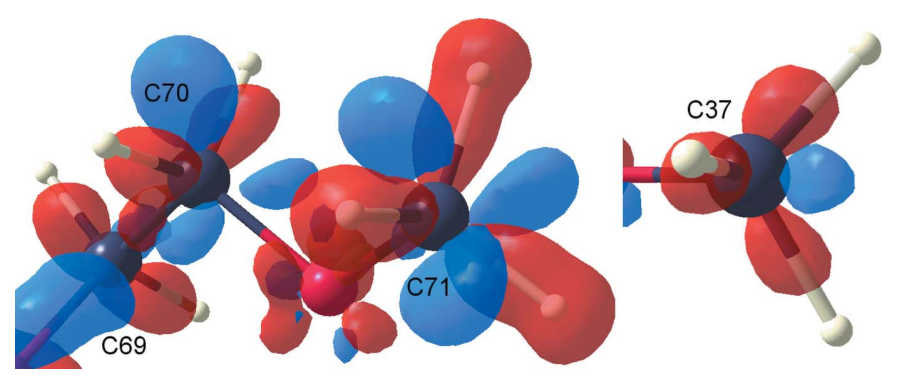

Figure 3

Difference density: experimental multipole refinement (EMR) minus invariom refinement (IR). Left: oxime chain (atoms C69, C70 and C71) and right: cladinose sugar (atom C37). Iso-surface values are 0.1 (blue) and -0.1 e $\AA^{-3}$ (red). Additional electron density in blue between bonds and missing electron density in red in the middle of the $\mathrm{C}-\mathrm{H}$ bonds indicate hydrogen disorder. disorder seems to be absent from a conventional IAM refinement of roxithromycin we will show that electrondensity validation is necessary to obtain a realistic and unbiased EDD.

It was shown before that hydrogen disorder can be partly modeled by the flexible spherical-harmonic density functions $\left(d_{\mathrm{lm}}\right)$ of the multipole model (Dittrich, Warren et al., 2009), while the harmonic approximation of displacement parameters is then not able to describe the ensemble of thermal motion and EDD. Nor are split positions a remedy when electron density and atomic motion are smeared over a larger region of space as in hydrogen disorder. Split positions only lead to an increased number of parameters, but not to a better model in terms of physical significance. In case such disorder occurs, a difference density exceeding the effects of hydrogen bonding and crystal field - which are usually of the order 0.25 e $\AA^{-3}$ (Spackman et al., 1999; Dittrich \& Spackman, 2007) - has previously been found (Dittrich, Warren et al., 2009). A difference density of EMR minus IR revealed disorder around the atoms C37, C69, C70 and C71, mainly affecting the $\mathrm{H}$ atoms attached. This is illustrated in Fig. 3, in which density differences are represented by two iso-surfaces which were generated using the program MOLISO (Hübschle \& Luger, 2006). Although the visible difference is accounted for by multipoles of $\mathrm{C}$ atoms, it is the ensemble of parameters that describes such features in a pseudoatom representation. Areas of additional electron density from experiment (EMR) are shown in blue; areas of lower electron density than in the invariom refinement (IR) are mapped in red. Bonding regions of $\mathrm{C}-\mathrm{H}$ bonds have a lower electron density in the EMR, while areas between two hydrogen positions have a higher density than in the IR. These features can be ascribed to disorder. The amount of disorder is smaller for the methoxy group of the $\mathrm{C} 37$ atom compared with the methoxy group of the C71 atom, as can be deduced from the different size of the iso-surfaces.

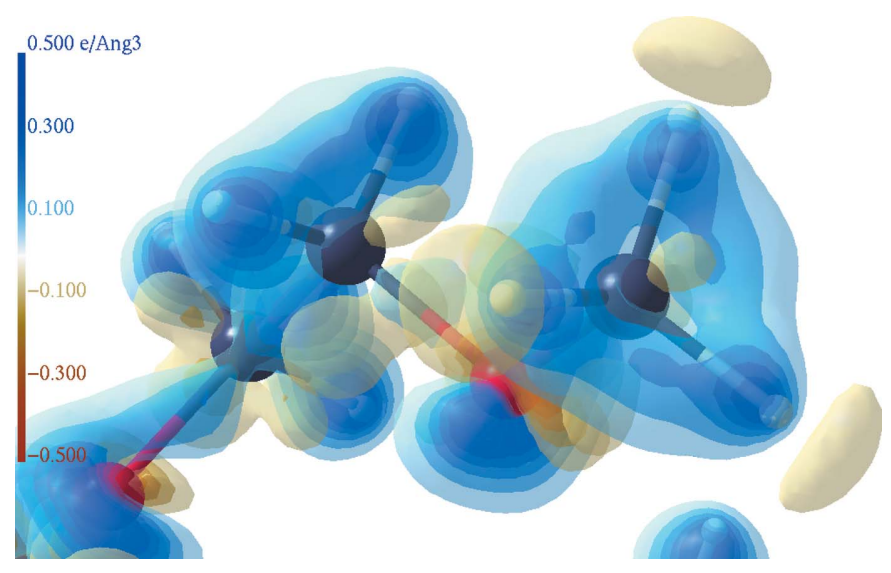

Figure 4

Three-dimensional deformation density of the disordered groups, illustrating $\mathrm{H}$-disorder. Iso-contours with a colour gradient from light

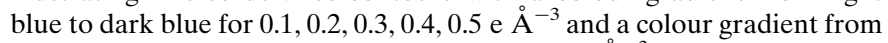
yellow to red for $-0.1,-0.2,-0.3,-0.4,-0.5$ e $\AA^{-3}$. 
Table 3

Atomic charges (in e) and volumes (in $\AA^{3}$ ) according to Bader's QTAIM for three different refinements of roxithromycin.

\begin{tabular}{|c|c|c|c|c|c|c|}
\hline \multirow[b]{2}{*}{ Atom } & \multicolumn{3}{|c|}{ Charges $Q$} & \multicolumn{3}{|l|}{$V_{\text {tot }}$} \\
\hline & IR & EMR & MMR & IR & EMR & MMR \\
\hline C37 & 0.26 & 0.11 & 0.28 & 10.3 & 11.7 & 10.6 \\
\hline C69 & 0.28 & 0.21 & 0.29 & 8.6 & 9.5 & 8.8 \\
\hline C70 & 0.31 & 0.23 & 0.31 & 8.5 & 10.1 & 8.7 \\
\hline C71 & 0.25 & 0.01 & 0.26 & 11.8 & 15.5 & 12.6 \\
\hline Н37 $A$ & 0.08 & 0.08 & 0.08 & 7.9 & 7.4 & 7.7 \\
\hline Н37B & 0.08 & 0.07 & 0.08 & 9.3 & 8.7 & 9.1 \\
\hline H37C & 0.08 & 0.08 & 0.08 & 8.9 & 8.2 & 8.6 \\
\hline H69A & 0.06 & 0.10 & 0.06 & 10.3 & 9.7 & 10.4 \\
\hline H69B & 0.05 & 0.09 & 0.06 & 9.1 & 8.3 & 8.9 \\
\hline $\mathrm{H} 70 A$ & 0.06 & 0.12 & 0.07 & 7.3 & 6.4 & 7.0 \\
\hline $\mathrm{H} 70 B$ & 0.05 & 0.11 & 0.06 & 7.6 & 6.9 & 7.3 \\
\hline $\mathrm{H} 71 A$ & 0.07 & 0.23 & 0.07 & 8.3 & 6.1 & 7.9 \\
\hline $\mathrm{H} 71 B$ & 0.08 & 0.23 & 0.08 & 7.9 & 6.2 & 7.7 \\
\hline $\mathrm{H} 71 C$ & 0.09 & 0.23 & 0.09 & 8.2 & 6.2 & 7.8 \\
\hline
\end{tabular}

Disorder can also be illustrated from the EMR alone by a three-dimensional static deformation density map in the region of atoms C37, C69, C70 and C71 and the attached $\mathrm{H}$ atoms. The shape of the deformation density of the terminal oxime-chain atom C71 is torus-like, as shown in Fig. 4. The coordination sphere of the above listed atoms is tetrahedral. Therefore, a three-dimensional representation is more complete and convincing than a two-dimensional contour-line plot. Hence, we chose to visualize the effect by a threedimensional deformation density with ten iso-surfaces using MOLISO (Hübschle \& Luger, 2006). An even more straightforward way to reveal hydrogen disorder is a topological analysis of $\mathrm{C}-\mathrm{H}$ bond-critical points $(\mathrm{BCP})$, using the XDPROP subprogram of XD (Koritsánszky et al., 2003). In the EMR significantly smaller values of $\rho\left(\mathbf{r}_{\mathrm{BCP}}\right)$ for bonds involved in disorder than expected from theory (or IR model) were found (supplementary information).

The observation of disorder led us to modify our experimental multipole model. To obtain a physically meaningful electron density model uninfluenced by disorder we generated a 'mixed model refinement' (MMR). In this model the C37, $\mathrm{C} 69, \mathrm{C} 70$ and $\mathrm{C} 71$ atoms, and attached disordered $\mathrm{H}$ atoms were kept at invariom-database values, whereas all other multipole parameters were refined in the same way as in the EMR. A similar procedure has recently been applied to paracetamol (Bak et al., 2009), in which dynamic rotational disorder of a methyl group has been discovered by careful analysis. In addition, positional parameters for non-H atoms were refined again. Figures of merit (FOM) for all three models are listed in Table 2. Owing to the refinement of positional parameters of non-H atoms in MMR, residuals improved when compared with EMR in which the atomic positions of IR were kept. It is also the reason for the higher number of parameters.

The qualitative characterization of disorder can be further substantiated. By calculating integrated atomic properties according to Baders quantum theory of atoms in molecules (QTAIM; Bader, 1990) the extent of bias can be quantified. Integrated atomic properties were calculated for all three refinement models using the program $X D P R O P$ and are listed in Table 3. The refined methoxy group bonded to the C71 atom is strongly affected in the EMR: C71 has almost no charge (0.01 e) and its attached hydrogen atoms $\mathrm{H} 71 A-C$ have a positive charge of 0.23 e per $\mathrm{H}$ atom. When kept at invariom database values $\mathrm{C} 71$ has, as expected for a methoxy $\mathrm{C}$ atom, a positive charge ( $0.26 \mathrm{e}$ in MMR and $0.25 \mathrm{e}$ in IR) while its attached $\mathrm{H}$ atoms $\mathrm{H} 71 A-C$ only have a charge of 0.08 e for both MMR and IR. Hence, in EMR the $\mathrm{C}$ atoms affected by disorder have a larger volume and correspondingly a more negative charge, while the $\mathrm{H}$ atoms have a lower volume and a more positive charge; the general trend of refined disordered $\mathrm{C}-\mathrm{H}$ groups is a shift of electron density from $\mathrm{H}$ to $\mathrm{C}$ atoms. Since an overall charge constraint is usually used in multipole refinements ('keep charge group 1' in $X D L S M$ ), such local bias can indirectly affect all refined multipole parameters. The analysis shows that ultimately only the MMR should be used for the purpose of analyzing the refined electron density and for calculating residual density, deformation densities, the molecular electrostatic potential and dipole moment.

\subsection{Hybrid scattering factors for $H$ atoms}

Proper modeling of hydrogen-atom scattering in multipole refinements recently attracted considerable interest (Hoser et al., 2009). The hitherto common treatment of $\mathrm{H}$ atoms involves refinement of only monopole and dipole populations, sometimes adding quadrupolar parameters for $\mathrm{H}$ atoms involved in hydrogen bonding. Here we chose to use 'hybrid' scattering factors for $\mathrm{H}$ atoms, where non-refined quadrupole, octupole and hexadecapole populations were kept at the invariom database values, whereas hydrogen monopoles and dipoles (and sometimes quadrupoles) were refined. We think this is an alternative to imposing a linear relation between the ratio of dipole and quadrupole populations (Abramov et al., 2000) for $\mathrm{H}$ atoms. A comparison of figures of merit indicates a marginally lower $R(F)$ value (0.0204 for hybrid scattering factors, 0.0205 for conventional scattering factors) and a better goodness-of-fit for hybrid $\mathrm{H}$-atom scattering factors (3.54 for hybrid scattering factors compared with 3.61 for conventional scattering factors). More importantly, a difference map between the density from a conventional treatment and from hybrid scattering factors for $\mathrm{H}$ atoms in terms of a positive and a negative iso-surface (Fig. 5, generated with the program MOLISO) reveals a systematic shift of additional electron density (blue isosurface) away from the carbon monopole towards the $\mathrm{H}$ atoms attached to them; areas of a depletion of electron density are depicted in red. This effect is especially pronounced for $R_{2} \mathrm{CH}_{2}$ and $R \mathrm{CH}_{3}$ groups and also leads to differences in integrated properties. Since the choice of limiting $l_{\max }$ for $\mathrm{H}$ atoms in conventional multipole refinements is only due to their limited scattering power, incorporating additional information on $\mathrm{H}$-atom scattering seems useful. We therefore think that it is appropriate to fix higher multipoles (up to hexadecapolar level) for $\mathrm{H}$ atoms to improve agreement with theoretical computations and to increase the reliability of experimental results in multipole refinements. An 
Table 4

Topological analysis of the hydrogen bonds in roxithromycin based on the MMR model.

$\epsilon$ is the bond ellipticity, $\rho\left(\mathbf{r}_{\mathrm{BCP}}\right)$ the electron density at the BCP in units of e $\AA^{-3}$ and $\nabla^{2} \rho\left(\mathbf{r}_{\mathrm{BCP}}\right)$ the Laplacian $\left(\mathrm{e} \AA^{-5}\right)$ of the electron density. Interatomic distances are given in $\AA$, angles in $\left({ }^{\circ}\right)$.

\begin{tabular}{|c|c|c|c|c|c|c|c|}
\hline$D-\mathrm{H} \cdots A$ & $D-\mathrm{H}$ & $\mathrm{H} \cdots A$ & $D \cdots A$ & $D-\mathrm{H} \cdots A$ & $\rho\left(\mathbf{r}_{\mathrm{BCP}}\right)$ & $\nabla^{2} \rho\left(\mathbf{r}_{\mathrm{BCP}}\right)$ & $\epsilon$ \\
\hline $\mathrm{O} 22-\mathrm{H} 22 \cdots \mathrm{O} 27$ & 0.96 & 1.89 & 2.83 & 163 & 0.19 & 2.2 & 0.07 \\
\hline $\mathrm{O} 23-\mathrm{H} 23 \cdots \mathrm{O} 80$ & 0.97 & 1.81 & 2.73 & 158 & 0.21 & 2.5 & 0.11 \\
\hline $\mathrm{O} 80-\mathrm{H} 80 A \cdots \mathrm{O} 26$ & 0.96 & 1.89 & 2.84 & 168 & 0.27 & 1.0 & 0.02 \\
\hline $\mathrm{O} 80-\mathrm{H} 80 B \cdots \mathrm{O} 21$ & 0.96 & 1.96 & 2.91 & 167 & 0.16 & 1.2 & 0.05 \\
\hline $\mathrm{O} 39-\mathrm{H} 39 \cdots \mathrm{N} 2^{\mathrm{i}}$ & 0.96 & 1.77 & 2.72 & 173 & 0.38 & 2.0 & 0.01 \\
\hline $\mathrm{O} 59-\mathrm{H} 59 \cdots \mathrm{O} 39^{\mathrm{ii}}$ & 0.96 & 2.00 & 2.94 & 164 & 0.11 & 2.3 & 0.18 \\
\hline
\end{tabular}

Symmetry codes: (i) $1-x, \frac{1}{2}+y, \frac{1}{2}-z$; (ii) $1-x, \frac{1}{2}+y, \frac{1}{2}-z$.

even better technical implementation would be to introduce restraints with a very low standard deviation for these parameters, as recommended earlier (Dittrich, Hübschle et al., 2009).

\subsection{Hydrogen bonding}

Atoms of the macrocycle are involved in one intramolecular hydrogen bond and two intermolecular bonds to other macrolide molecules. In addition, the solvent water molecule acts once as an acceptor (for $\mathrm{O} 23-\mathrm{H}$ ) and twice as a donor to bridge the keto oxygen $\mathrm{O} 26$ with the carbonyl oxygen $\mathrm{O} 21$. Details of these and two further intermolecular hydrogen bonds are given in Table 4. The conformation of the 14membered lactone ring is virtually identical to that found in the erythromycin derivative VT108 determined earlier (Luger et al., 1991). Such conformational stability of the macrocycle has already been noted by Bachet et al. (1988). Concerning

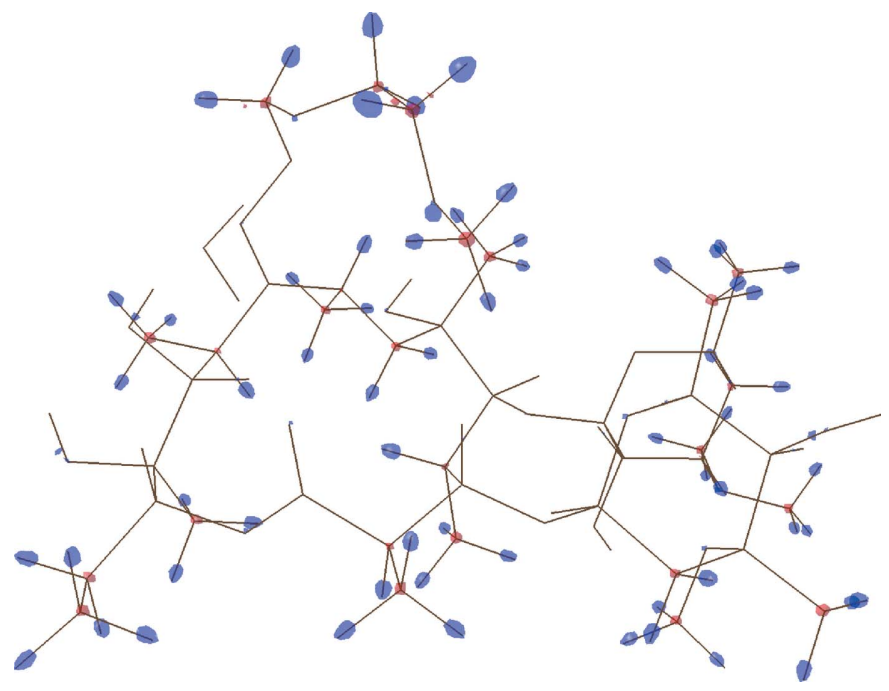

Figure 5

Difference density: EMR minus a classical multipole refinement. In the latter only monopole and dipole populations were refined for $\mathrm{H}$ atoms, whereas the EMR includes database multipoles with $l_{\max } \geq 2$ for $\mathrm{H}$ atoms up to the hexadecapolar level. Systematic additional electron density appears at hydrogen sites, while there is less electron density at $\mathrm{C}$-atom

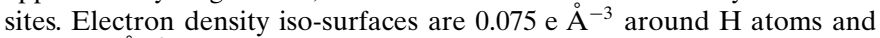
-0.075 e $\AA^{-3}$ around $\mathrm{C}$ atoms. their conformation, the cladinose sugar has the ${ }^{1} C_{4}(\mathrm{~L})$ form and L-ribo configuration (Hofheinz et al., 1962), while desosamine, belonging to the D series (Woo et al., 1992), is in the ${ }^{4} C_{1}$ (D) form. The glycosidic linkages to the macrocycle are $\alpha$ for cladinose and $\beta$ for desosamine. The electron density $(\rho \mathbf{r})$ at the bond critical point $(\mathrm{BCP})$ is a measure of the character of a chemical bond, which includes $\mathrm{H}$ bridges (Bader, 1990). Since covalent bonding has been extensively studied using topological analysis of experimental charge densities over the last years, and since hydrogen bonding has a strong influence on ESP and dipole moment, we will focus exclusively on hydrogen bonding topology here. Further, bondtopological parameters of covalent bonds can be found in the supplementary information.

A topological analysis of the electron density of hydrogen bonds was carried out for all three refinements. Despite differences in the ED modeling of some $\mathrm{H}$ atoms detailed in the previous section, very similar values for $\rho\left(\mathbf{r}_{\mathrm{BCP}}\right)$ for EMR and MMR (both using hybrid scattering factors) were

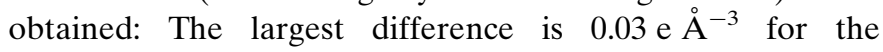
$\mathrm{H} 80 A \cdots \mathrm{O} 26$ bond. Regarding only the MMR, the strongest bond - indicated by the highest value of $\rho\left(\mathbf{r}_{\mathrm{BCP}}\right)=0.39$ e $\AA^{-3}$ is the intermolecular $\mathrm{H} 39-\mathrm{N} 2$ bond. The H23 . OO 80 bond, which has an almost similar $D-A$ bond length, is nevertheless much weaker, with $\rho\left(\mathbf{r}_{\mathrm{BCP}}\right)$ being only 0.21 e $\AA^{-3}$ in this case. The high value of density at the BCP of the hydrogen bond $\mathrm{O} 39-\mathrm{H} 39 \cdots \mathrm{N} 2^{\mathrm{i}}$ suggests a rather strong interaction. The high value of density at the $\mathrm{BCP}$ can be understood by taking into account the hydrogen-bond pattern around O39 that accepts the H59 atom in addition to donating H39. The values for all hydrogen bonds are given in Tables 4 and 5 .

For the MMR the bond-critical point properties and the donor-aceptor distances have also been evaluated by an empirical relationship based on the Laplacian of the electron density (Abramov, 1997) for $G\left(\mathbf{r}_{\mathrm{BCP}}\right)$. Additionally a fit to results from experimental charge density studies (Espinosa $e t$ al., 1998) was used to calculate $V\left(\mathbf{r}_{\mathrm{BCP}}\right)$ and hydrogen-bond energies $E_{\mathrm{HB}}$. These are given in Table 5. Since two hydrogen

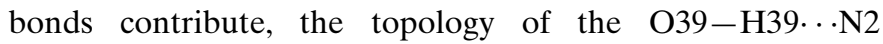
hydrogen bond might lead to an overestimation of the $E_{\mathrm{HB}}($ Espinosa) hydrogen-bond energy.

\subsection{Electrostatic potential}

To visualize the effect of the conformational disorder on the ESP included in the EMR we calculated the EMR's electrostatic potential ( $\mathrm{Su} \&$ Coppens, 1992) and mapped it on the EMR 'van der Waals' iso-surface of electron density

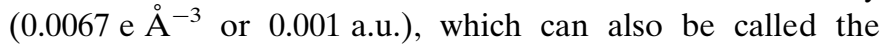
molecular surface, using MOLISO. Owing to the size of the molecule we will first focus on one methoxy function by comparing the resulting local ESP of the invariom approach (IR) with the experimental one (EMR). Fig. 6 shows a diverging potential in the regions next to hydrogen positions 
Table 5

Electron density at BCPs $\rho\left(\mathbf{r}_{\mathrm{BCP}}\right)$ of hydrogen bonds in e $\AA^{-3}$ (columns 24), kinetic energy density (column 5), potential energy density (column 6) and hydrogen bond energy in $\mathrm{kJ} \mathrm{mol}^{-1}$.

\begin{tabular}{lllllll}
\hline $\mathrm{H} \cdots A$ & IR & EMR & MMR & $G\left(\mathbf{r}_{\mathrm{BCP}}\right)$ & $V\left(\mathbf{r}_{\mathrm{BCP}}\right)$ & $E_{\mathrm{HB}}($ Espinosa $)$ \\
\hline $\mathrm{H} 22 \cdots \mathrm{O} 27$ & 0.16 & 0.20 & 0.19 & 59.6 & -59.3 & -29.6 \\
$\mathrm{H} 23 \cdots \mathrm{O} 80$ & 0.22 & 0.21 & 0.21 & 68.6 & -69.1 & -34.6 \\
$\mathrm{H} 80 A \cdots \mathrm{O} 26$ & 0.18 & 0.24 & 0.27 & 53.4 & -79.6 & -39.8 \\
$\mathrm{H} 80 B \cdots \mathrm{O} 21$ & 0.14 & 0.15 & 0.16 & 36.5 & -40.4 & -20.2 \\
$\mathrm{H} 39 \cdots \mathrm{N}^{\mathrm{i}}$ & 0.27 & 0.39 & 0.38 & 98.7 & -142.9 & -71.4 \\
$\mathrm{H} 59 \cdots \mathrm{O} 39^{\text {ii }}$ & 0.13 & 0.11 & 0.11 & 49.7 & -36.7 & -18.3 \\
\hline
\end{tabular}

Symmetry codes: (i) $1-x, \frac{1}{2}+y, \frac{1}{2}-z$; (ii) $1-x, \frac{1}{2}+y, \frac{1}{2}-z$.

of the disordered methoxy group (C71). While the surface is colored in blue for EMR indicating a strongly positive region, the surface calculated from the invariom refinement (IR) is colored in grey, indicating a neutral or only slightly positive potential. This illustrates that one has to be very careful not to interpret features in the potential that might be due to possibly overlooked - hydrogen disorder. Regarding the unbiased electrostatic potential, Fig. 7 represents the ESP calculated from the MMR model, again mapped on the 'van

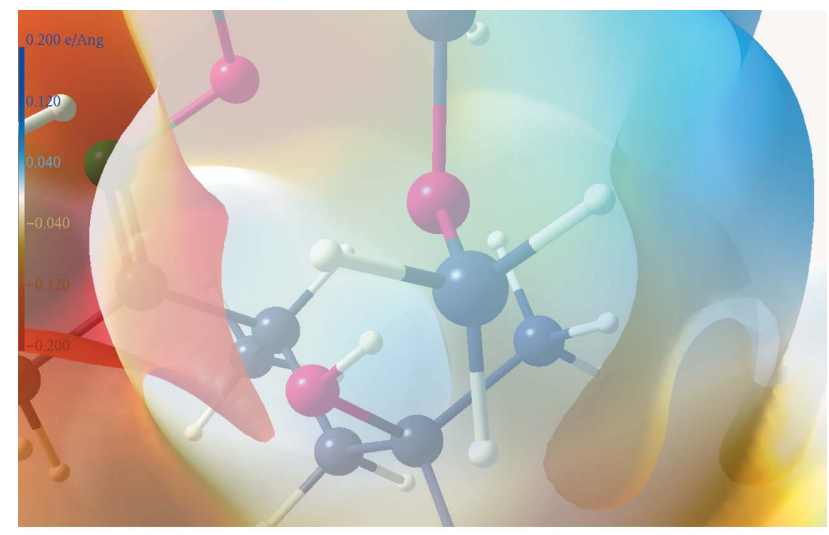

(a)

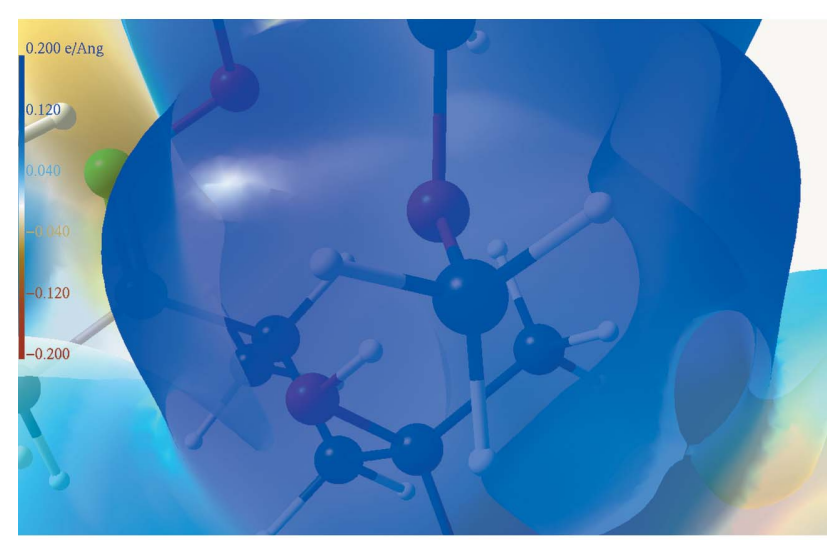

Figure 6

(b)

Electrostatic potential mapped on the molecular surface in the area of the methoxy group (atom C71). (a) Invariom refinement (IR) and (b) EMR model. While the IR result appears to be realistic, the disorder affecting some multipole parameters in the EMR leads to unrealistic polarizations in this region. der Waals' iso-surface of electron density. It shows a comparably high negative potential at the desosamine sugar (lower picture, orange/red colored on the left). We ascribe this negative potential to the hydrogen-bonding pattern (see $\$ 4.3$ ). Whereas Fig. 6 contains the effect of disorder bias on the potential, Fig. 7 represents probably the more realistic situation of an increase in both more positive and negative features stabilized by the surrounding roxithromycin molecules in the crystal. Such effects of hydrogen bonding and the crystal field would not be included in a prediction based on invariomdatabase density parameters and their derived ESP (shown in Fig. 8). Nevertheless, the electrostatic potential calculated from the invariom database and the mixed model refinement agree rather well qualitatively, in particular considering the

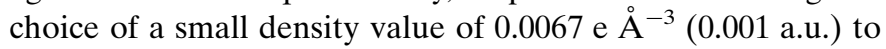
generate the molecular surface enhances even small differences.

Roxithromycin is a rather large molecule and a visual comparison of the potential can be cumbersome. Therefore, following the procedure of Politzer and coworkers (Politzer et

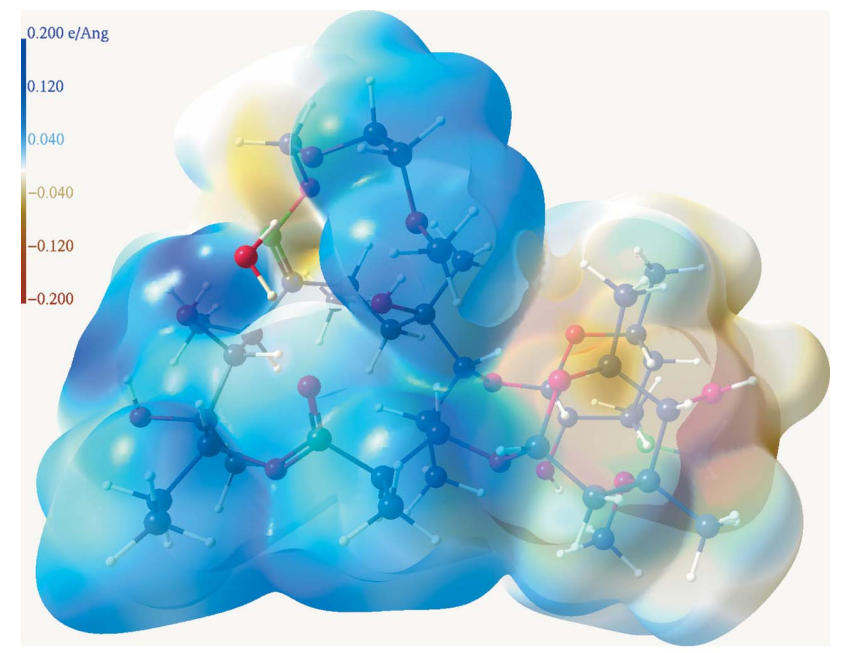

(a)

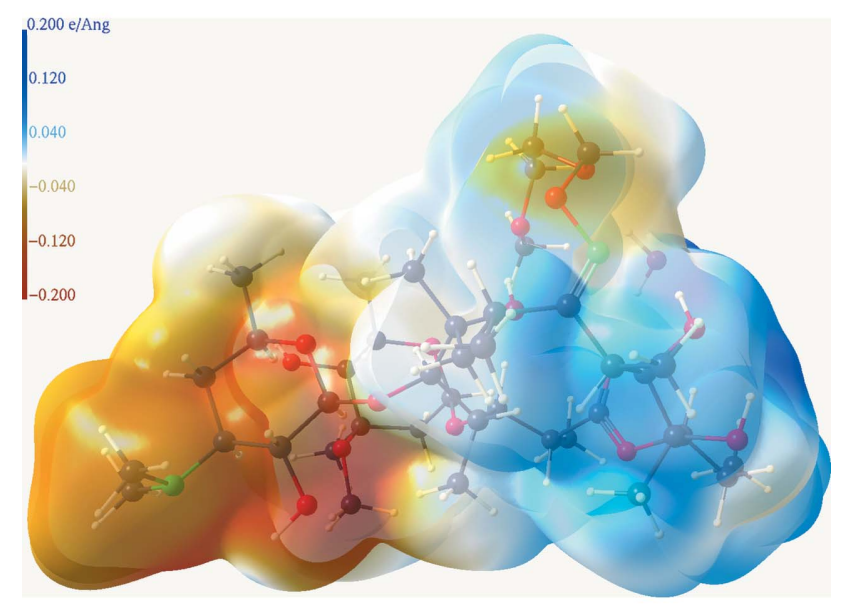

(b)

Figure 7

Front and back view of the electrostatic potential mapped on the van der Waals surface for the mixed model refinement (MMR). The potential of the solvent water molecule was excluded from the calculation and is not shown. 
Table 6

Politzer analysis of the ESP mapped onto the molecular surface of roxithromycin.

\begin{tabular}{lrrr}
\hline Property & \multicolumn{1}{c}{ IR } & \multicolumn{1}{c}{ EMR } & \multicolumn{1}{c}{ MMR } \\
\hline $\bar{V}_{S}^{+}\left(\mathrm{e} \AA^{-1}\right)$ & 0.0283 & 0.0510 & 0.0440 \\
$\bar{V}_{S}^{-}\left(\mathrm{e} \AA^{-1}\right)$ & -0.0540 & -0.0665 & -0.0612 \\
$\Pi_{\left(\mathrm{e} \AA^{-1}\right)}$ & 0.0424 & 0.0592 & 0.0522 \\
$\sigma_{\text {tot }}^{2}\left(\mathrm{e}^{2} \AA^{-2}\right)$ & 0.0058 & 0.0088 & 0.0089 \\
\hline
\end{tabular}

al., 2001) we performed a quantitative analysis of the ESP. Such an analysis has been implemented in the program MOLISO and was first adapted in the charge-density study of the nucleoside thymidine (Hübschle et al., 2008). The positive, negative and overall average potential values $V_{S}^{+}, V_{S}^{-}$and $V_{S}$ on the surface are calculated as given in (1)-(3), where $n$ and $m$ are the number of grid points with positive and negative potential

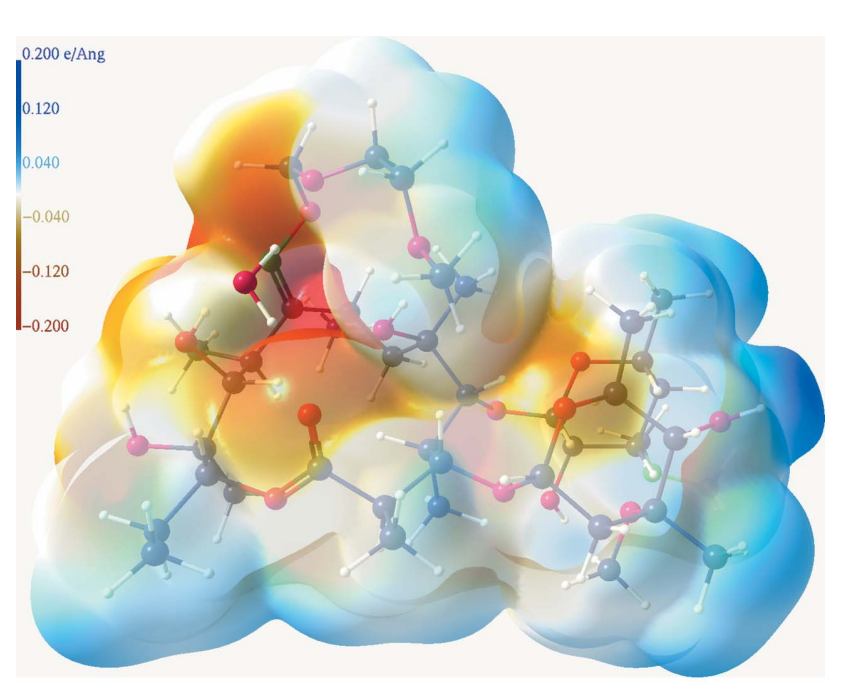

(a)

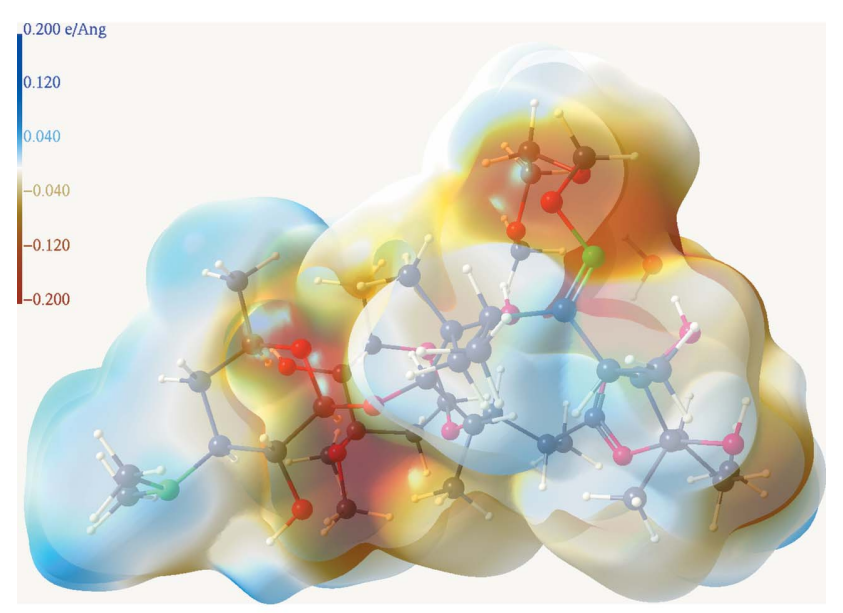

(b)

Figure 8

Front and back view of the electrostatic potential mapped on the van der Waals surface for the invariom refinement (IR). The potential of the solvent water molecule was excluded from the calculation and is not shown.

$$
\begin{aligned}
& \bar{V}_{S}^{+}=\frac{1}{n} \sum_{i=1}^{n} V_{S}^{+}\left(\mathbf{r}_{i}\right), \\
& \bar{V}_{S}^{-}=\frac{1}{m} \sum_{i=1}^{m} V_{S}^{-}\left(\mathbf{r}_{i}\right), \\
& \bar{V}_{S}=\frac{1}{m+n}\left[\sum_{i=1}^{n} V_{S}^{+}\left(\mathbf{r}_{i}\right)+\sum_{i=1}^{m} V_{S}^{-}\left(\mathbf{r}_{i}\right)\right] .
\end{aligned}
$$

The average deviation from the overall potential on the surface is $\Pi$

$$
\Pi=\frac{1}{m+n} \sum_{i=1}^{m+n}\left|V_{S}\left(\mathbf{r}_{i}\right)-\bar{V}_{S}\right| .
$$

The positive, negative and total variances of the surface potential are

$\sigma_{\mathrm{tot}}^{2}=\sigma_{+}^{2}+\sigma_{-}^{2}=\frac{1}{n} \sum_{i=1}^{n}\left[V_{S}^{+}\left(\mathbf{r}_{i}\right)-\bar{V}_{S}^{+}\right]^{2}+\frac{1}{m} \sum_{i=1}^{m}\left[V_{S}^{-}\left(\mathbf{r}_{i}\right)-\bar{V}_{S}^{-}\right]^{2}$.

We can see from values given in Table 6 that the EMR leads to the highest spread of $\bar{V}_{S}^{+} / \bar{V}_{S}^{-}$values most likely due to disorder bias in this multipole model. The $\bar{V}_{S}^{+} / \bar{V}_{S}^{-}$values of the MMR are more similar to the EMR. In the IR an incorporation of hydrogen bonding and crystal effects is missing, which manifests itself in lower values for $\bar{V}_{S}^{+} / \bar{V}_{S}^{-}$in the IR in comparison to the MMR.

\subsection{Residual density analysis}

Recently a quantitative analysis of the residual density (RDA) was introduced (Meindl \& Henn, 2008), where the residual electron density not taken into account by a particular density model is analyzed. RDA allows the detection of problems in a density model or the comparison of the quality of different models. The residual density of MMR was calculated on a three-dimensional grid spanning the whole unit cell for such an evaluation. The total amount of residual density present was characterized by a summation over the modulus of the residual density values times the unit-cell volume, which yielded 41.3 gross residual electrons. The range of residual

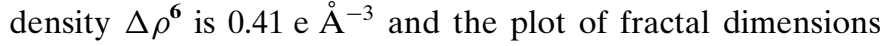
versus residual density is almost mirror-symmetric to the vertical axis on position zero (see Fig. 9), a measure of a good refinement model. Residual density plots for the other models considered contain more features and are given in the supplementary information.

\subsection{Dipole moments}

The dipole moment of a molecule in the crystal lattice has been shown to be frequently enhanced when compared to results from computations of an isolated molecule in the gas phase with an identical geometry. A recent mini-review evaluated and summarized a considerable number of studies (Spackman et al., 2007), but also highlighted cases where an

$\overline{{ }^{6} \Delta \rho=\rho_{\max }-\rho_{\min }}$. 
Table 7

Dipole moments of theoretical computations and refinement models of roxithromycin.

'Theory' refers to a single-point energy calculation with the method/basis set B3LYP/D95++(3df,3pd) using the geometry of the MMR. The total dipole moment is given in Debye (D).

\begin{tabular}{llrrr}
\hline Dipole moment & Theory & IR & \multicolumn{1}{c}{ EMR } & \multicolumn{1}{c}{ MMR } \\
\hline$\mu_{X}$ & 7.1 & 6.4 & $2.3(9)$ & $-3.2(8)$ \\
$\mu_{Y}$ & 1.7 & 1.5 & $13.1(9)$ & $12.9(10)$ \\
$\mu_{Z}$ & 6.1 & 7.6 & $-18.0(18)$ & $-15.2(18)$ \\
$\mu_{\text {total }}$ & 9.5 & 10.1 & $22.4(16)$ & $20.3(15)$ \\
\hline
\end{tabular}

unrealistic enhancement in the molecular dipole moment has been obtained from modeling X-ray diffraction data. To obtain the dipole moment of an isolated molecule of roxithromycin in the gas phase, a single-point energy calculation using the geometry of MMR was performed. ${ }^{7}$ In contrast to the rapid summation of pseudoatom components after multipole or invariom refinement taking only seconds on a current computer, even a high-level single-point calculation not to mention a geometry optimization - can be time consuming for a comparably large molecule like roxithromycin. As is shown in Table 7, invariom refinement can nevertheless provide a good estimate of the theoretical value, which is in good agreement both in magnitude and the individual components $\mu_{X}, \mu_{Y}$ and $\mu_{Z}$.

With changes in the in-crystal dipole moment of the order $10 \%$ between EMR and MMR, we find that different treatment of the hydrogen disorder clearly affects the molecular dipole moment. We think that disorder treatment makes the experimental estimate more realistic. However, the difference between both theoretical approaches (theory and IR) and the experimental results (EMR and MMR) is even higher - the total dipole moment more than doubles after refinement. All values including the individual components are listed in Table 7. It is conceivable that a doubling of the value is caused by crystal-field effects, but the result should probably be treated with caution and conclusive evidence cannot be provided at this point. Future theoretical calculations should asses whether the large enhancement is indeed caused by the crystal field or is an artefact.

\section{Conclusion}

Complete and redundant diffraction data for the antibiotic roxithromycin have been collected with synchrotron radiation at low temperature to a high resolution. On first sight the roxithromycin structure does not contain disordered domains. Hydrogen disorder was only revealed by calculating the difference density between theoretical prediction and experimental refinement results and could therefore have escaped the attention of the crystallographer. Hydrogen disorder was found to be of considerable importance in charge density refinements, since it can lead to a false electron density

\footnotetext{
${ }^{7}$ The same level of theory currently used to calculate invariom fragments was employed, which is the B3LYP functional with the D95++(3df,3pd) basis set.
}

$\rho(\mathbf{r})$ and to bias in integrated atomic properties (like $V$ and $Q)$, in the electrostatic potential $V(\mathbf{r})$ and the dipole moment.

Experimental diffraction data were evaluated by three multipole models. Hydrogen-atom scattering was described by combining theory and experiment in the form of a hybrid scattering factor. This procedure does not necessarily improve the fit to the experimental data when compared with conventional approaches in charge density research, but eliminates correlations between monopole and higher multipole populations of $\mathrm{H}$ atoms. Hydrogen monopole populations in turn can bias parameters of all other atoms via the overall charge constraint. Using hybrid scattering factors is facilitated by invariom transfer and increases agreement with theory. Hence, for future studies, independent of the presence of disorder, we recommend the use of fixed bond-directed quadrupole, octupole and hexadecapole parameters for $\mathrm{H}$ atoms from the invariom database, while refining only their monopole and dipole populations. When estimated hydrogen ADPs are available, quadrupoles might also be refined in the presence of hydrogen bonds. Together with restricted $\kappa$ parameters, the reliability of results from charge density modeling can be enhanced this way, which is especially important for larger molecules.

Our primary objective, to extract a physically meaningful electron density for roxithromycin molecules in the crystal and to calculate accurate derived properties such as the ESP, was achieved in a mixed model refinement where multipoles of only the disordered groups were kept at invariom database values, providing a generally viable modeling procedure in the presence of disordered groups.

Funding for this work within the Deutsche Forschungsgemeinschaft (DFG), grants DI 921/3-1 and Lu222/29-2 (SPP 1178), is greatfully acknowledged. We thank Dr K. Meindl and Dr J. Henn for an executable for performing residual density analysis and Dr F. P. A. Fabbiani for reading the manuscript and corrections therein.

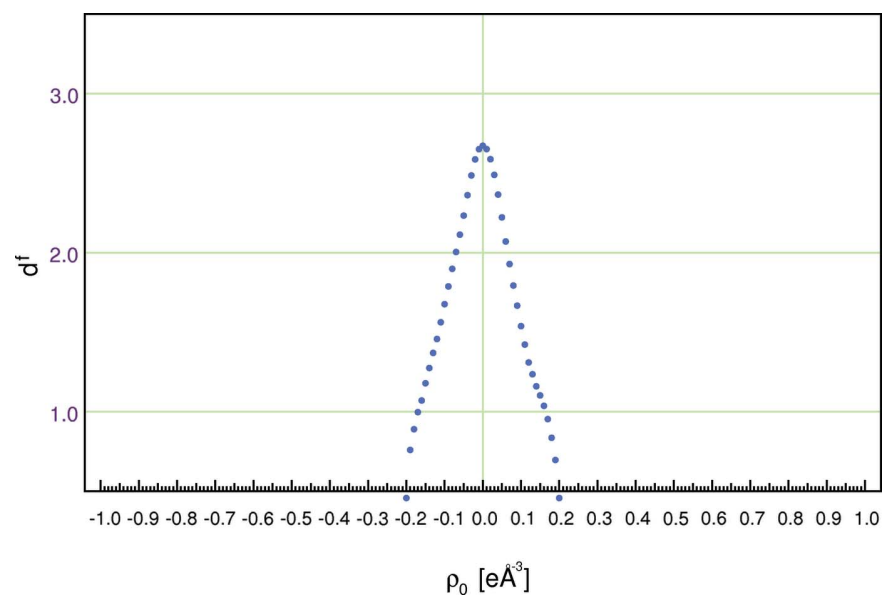

Figure 9

Meindl plot: Fractal dimensions $\left(d_{f}\right)$ versus residual density $\left(\rho_{0}\right)$ for the MM refinement model of roxithromycin. 


\section{References}

Abramov, Yu. A. (1997). Acta Cryst. A53, 264-272.

Abramov, Y. A., Volkov, A. V. \& Coppens, P. (1999). Chem. Phys. Lett. 311, 81-86.

Abramov, Y. A., Volkov, A., Wu, G. \& Coppens, P. (2000). J. Phys. Chem. B, 104, 2183-2188.

Allen, F. H. \& Bruno, I. J. (2010). Acta Cryst. B66, 380-386.

Bachet, B., Brassy, C. \& Mornon, J.-P. (1988). Acta Cryst. C44, 112116.

Bader, R. F. W. (1990). Atoms in Molecules: A Quantum Theory. Oxford: Clarendon Press.

Bąk, J. M., Dominiak, P. M., Wilson, C. C. \& Woźniak, K. (2009). Acta Cryst. A65, 490-500.

Burnett, M. N. \& Johnson, C. K. (1996). ORTEPIII, Report ORNL6895. Oak Ridge National Laboratory, Tennessee, USA.

Coppens, P. (2005). Angew. Chem. Int. Ed. 44, 6810-6811.

Coppens, P. \& Volkov, A. (2004). Acta Cryst. A60, 357-364.

Dittrich, B., Hübschle, C. B., Holstein, J. J. \& Fabbiani, F. P. A. (2009). J. Appl. Cryst. 42, 1110-1121.

Dittrich, B., Hübschle, C. B., Luger, P. \& Spackman, M. A. (2006). Acta Cryst. D62, 1325-1335.

Dittrich, B., McKinnon, J. J. \& Warren, J. E. (2008). Acta Cryst. B64, 750-759.

Dittrich, B., Munshi, P. \& Spackman, M. A. (2007). Acta Cryst. B63, 505-509.

Dittrich, B. \& Spackman, M. A. (2007). Acta Cryst. A63, 426-436.

Dittrich, B., Warren, J. E., Fabbiani, F. P. A., Morgenroth, W. \& Corry, B. (2009). Phys. Chem. Chem. Phys. 11, 2601-2609.

Dominiak, P. M., Volkov, A., Li, X., Messerschmidt, M. \& Coppens, P. (2007). J. Chem. Theory Comput. 2, 232-247.

Espinosa, E., Molins, E. \& Lecomte, C. (1998). Chem. Phys. Lett. 285, 170-173.

Gatti, C., Saunders, V. R. \& Roetti, C. (1994). J. Chem. Phys. 101, 10686-10696.

Grabowsky, S., Kalinowski, R., Weber, M., Förster, D., Paulmann, C. \& Luger, P. (2009). Acta Cryst. B65, 488-501.

Hansen, N. K. \& Coppens, P. (1978). Acta Cryst. A34, 909-921.

Harms, J., Schluenzen, F., Zarivach, R., Bashan, A., Gat, S., Agmon, I., Bartels, H., Franceschi, F. \& Yonath, A. (2001). Cell, 107, 679688.

Harris, D. R., McGeachin, S. G. \& Mills, H. H. (1965). Tetrahedron Lett. 11, 679-685.

Hirshfeld, F. L. (1976). Acta Cryst. A32, 239-244.

Hofheinz, W., Griesbach, H. \& Friebolin, H. (1962). Tetrahedron, 18, $1265-1274$.

Hoser, A. A., Dominiak, P. M. \& Woźniak, K. (2009). Acta Cryst. A65, $300-311$.
Hübschle, C. B., Dittrich, B., Grabowsky, S., Messerschmidt, M. \& Luger, P. (2008). Acta Cryst. B64, 363-374.

Hübschle, C. B. \& Luger, P. (2006). J. Appl. Cryst. 39, 901-904.

Hübschle, C. B., Luger, P. \& Dittrich, B. (2007). J. Appl. Cryst. 40, 623-627.

Johnas, S. K. J., Dittrich, B., Meents, A., Messerschmidt, M. \& Weckert, E. F. (2009). Acta Cryst. D65, 284-293.

Kabsch, W. (1993). J. Appl. Cryst. 26, 795-800.

Koritsánszky, T., Richter, T., Macchi, P., Volkov, A., Gatti, C., Howard, S., Mallinson, P. R., Farrugia, L., Su, Z. W. \& Hansen, N. K. (2003). XD. Technical Report, Freie Universität Berlin, Germany.

Luger, P. (2007). Org. Biomol. Chem. 5, 2529-2540.

Luger, P., Prox, A. \& Woitun, E. (1991). Acta Cryst. C47, 19481952.

Madsen, A. Ø. (2006). J. Appl. Cryst. 39, 757-758.

Madsen, A. Ø., Sørensen, H. O., Flensburg, C., Stewart, R. F. \& Larsen, S. (2004). Acta Cryst. A60, 550-561.

Meindl, K. \& Henn, J. (2008). Acta Cryst. A64, 404-418.

Munshi, P., Madsen, A. Ø., Spackman, M. A., Larsen, S. \& Destro, R. (2008). Acta Cryst. A64, 465-475.

Politzer, P., Murray, J. S. \& Preralta-Inga, Z. (2001). Int. J. Quantum Chem. 85, 676-684.

Rödel, E., Messerschmidt, M., Dittrich, B. \& Luger, P. (2006). Org. Biomol. Chem. 4, 475-481.

Roversi, P. \& Destro, R. (2004). Chem. Phys. Lett. 386, 472-478.

Schlünzen, F., Zarivach, R., Harms, J., Bashan, A., Tocilj, A., Albrecht, R., Yonath, A. \& Franceschi, F. (2001). Nature, 321, 814-821.

Schomaker, V. \& Trueblood, K. N. (1968). Acta Cryst. B24, 63-76.

Sheldrick, G. M. (2008). Acta Cryst. A64, 112-122.

Spackman, M. A., Byrom, P. G., Alfredsson, M. \& Hermansson, K. (1999). Acta Cryst. A55, 30-47.

Spackman, M. A., Munshi, P. \& Dittrich, B. (2007). ChemPhysChem, 8, 2051-2063.

Spek, A. L. (2003). J. Appl. Cryst. 36, 7-13.

Su, Z. \& Coppens, P. (1992). Acta Cryst. A48, 188-197.

Tu, D., Blaha, G., Moore, P. B. \& Steitz, T. A. (2005). Cell, 121, $257-$ 270.

Volkov, A., Abramov, Y. A. \& Coppens, P. (2001). Acta Cryst. A57, 272-282.

Volkov, A., Messerschmidt, M. \& Coppens, P. (2007). Acta Cryst. D63, 160-170.

Whitten, A. E. \& Spackman, M. A. (2006). Acta Cryst. B62, 875888.

Woo, J.-T., Shinohara, C., Sakai, K., Hasumi, K. \& Endo, A. (1992). Eur. J. Biochem. 207, 383-389.

Zarychta, B., Pichon-Pesme, V., Guillot, B., Lecomte, C. \& Jelsch, C. (2007). Acta Cryst. A63, 108-125. 